\title{
Democracy made by the USA? Ein Blick auf die Parteienförderpraxis von US-Akteuren in jungen Demokratien
}

\section{Einmischung findet statt}

Die Demonstrations- und Revolutionswelle in Nordafrika und der arabischen Welt schwemmte eine Vielzahl externer Demokratieförderer in die neuen Transitionsstaaten. Ein bunter Strauß an internationalen Akteuren engagiert sich seither in Ägypten, Libyen, Tunesien und jüngst auch im Falle Syriens sowie darüber hinaus weltweit in den häufig als junge Demokratien gebündelten Ländern. Vor Ort begleiten sie den Übergang vom autoritären zum demokratischen System, beraten beim Aufbau politischer Institutionen und fördern oppositionelle Bewegungen. Im Fokus dieses Essays stehen die staatlichen und nicht-staatlichen US-amerikanischen Akteure wie das International Republican Institute (IRI) und das National Democratic Institute (NDI), die den beiden großen amerikanischen Parteien nahestehen, die U.S. Agency for International Development (USAID) oder das vom US-Kongress finanzierte United States Institute of Peace (USIP) und ihre Förderpraxis. ${ }^{1}$ Vor allem die Unterstützung politischer Parteien steht in den letzten zehn Jahren im Mittelpunkt der Demokratieförderung - was angesichts der zentralen Rolle, die den politischen Parteien im Prozess der Demokratisierung zukommt, auch nicht verwundert. Dabei ist zu beobachten, ${ }^{2}$ dass die Förderpraxis der US-Akteure zwar stringent durchgeplant erscheint - Leitlinien und Förderansätze liegen vor -, jedoch kaum Rücksicht genommen wird auf die Prozesshaftigkeit der demokratischen Transition und der Parteienentwicklung. Gleichzeitig mangelt es deutlich an langfristigen, nachhaltigen Kooperationen. $\mathrm{Zu}$ oft stehen die bloße Finanzierung von

1 Darüber hinaus findet man im Feld der Demokratie- und Parteienförderung eine Vielzahl weiterer internationaler Akteure, beispielsweise die deutschen parteinahen Stiftungen oder internationale Mehrparteieninstitute. Vergleiche hierzu auch Übersicht 1.

2 Die Verfasserin rekurriert hier auf eigene Forschungsaufenthalte und Interviewführung zur Parteienförderung in Kenia und Südafrika. Gespräche wurden vor Ort geführt mit Repräsentanten der von deutschen und US-Akteuren geförderten Oppositions- und Regierungsparteien sowie mit Wissenschaftlern und Experten des Bereichs Demokratie- und Parteienförderung. Ausführlicher dazu Weissenbach 2012. 
Förderpartnern und eine kurzfristige Unterstützung des Campaignings ausgewählter Parteien in Wahlzeiten im Vordergrund der Förderung.

Wie sensibel der Bereich der Parteienförderung ist, zeigen die Reaktionen der politischen Entscheidungsträger in Ägypten, welche die Parteienförderung der internationalen Akteure als Einmischung in innenpolitische Angelegenheiten einstufen: Anfang 2012 wurden in Ägypten 17 internationale Nichtregierungsorganisationen, darunter zwei Mitarbeiter der deutschen parteinahen Konrad-AdenauerStiftung sowie 19 US-Bürger, wegen des Vorwurfs fehlender Genehmigungen und der illegalen Finanzierung aus dem Ausland angeklagt (Wegerhoff 2012; o. A. 2012).

Diese Sensibilität ist der Grund dafür, dass das amerikanische Institut USIP die Förderung syrischer oppositioneller Kräfte nicht vor Ort durchführt, sondern aus dem Exil in Berlin. Gemeinsam mit der deutschen Stiftung Wissenschaft und Polititk (SWP) finanziert sie das sogenannte Projekt Day After, in dessen Rahmen regelmäßig oppositionelle syrische Kräfte zusammenkommen. Im Rahmen dieser Diskussionsplattform zwischen Ex-Generälen, Wirtschafts- und Justizvertretern und Repräsentanten aller Ethnien und Konfessionen wird hier beraten, wie der Übergang zu einem demokratischen System erfolgen und eine neue Verfassung aussehen kann (Lau 2012).

Dieser erste Blick auf die starke Präsenz von US-Akteuren im Feld der Demokratie- und Parteienförderung zeigt ganz deutlich: Einmischung in den Demokratisierungsprozess findet statt. Wer aber sind die internationalen Akteure der Demokratie- und Parteienförderung? Und wo in diesem Akteursspektrum positionieren sich die US-amerikanischen Organisationen? Auf welche Art und Weise laufen ihre Fördermaßnahmen ab und inwieweit liegen ihren Maßnahmen länderspezifische Konzepte und durchdachte Strategien zugrunde?

$\mathrm{Zu}$ diesen Fragestellungen, die das vorliegende Essay strukturieren, finden sich im Feld der Internationalen Beziehungen und der Entwicklungspolitik an der Schnittstelle von Transitions- und Demokratisierungsforschung sowie der internationalen Parteienforschung bislang nur wenige Analysen. 


\section{Von der Demokratie- zur Parteienförderung}

Dass politische Parteien und oppositionelle Bewegungen im Konsolidierungsprozess junger Demokratien eine entscheidende Rolle spielen, darüber ist sich die internationale Parteien- und Transitionsforschung weitgehend einig. ${ }^{3}$ Vor allem nachdem Thomas Carothers (2002) seine viel diskutierte und zitierte Kritik am „Transitionsparadigma" vorbrachte, rückte auch der Aspekt der Förderung von Parteien im Demokratisierungsprozess stärker in den politikwissenschaftlichen Fokus. Er zeigte unter Rückgriff auf die US-Demokratisierungshilfe Defizite der Förderung auf und plädierte für eine stärkere Forcierung der Parteienförderung. Auch Peter Burnell befasste sich 2006 in seinem Sammelband Globalising Democracy: Party Politics in Emerging Democracies mit dem Zusammenwirken von Globalisierung und Parteienentwicklung. Die erste Monografie, die einen Überblick über die Akteure der Parteienförderung, ihre Förderansätze und Instrumente sowie praktische Defizite und Verbesserungsvorschläge aufzeigt, legte Carothers mit Confronting the Weakest Link: Aiding Political Parties in New Democracies (2006) nach. In der Konsequenz bildete sich eine neue Forschungsnische der internationalen Entwicklungspolitik an der Schnittstelle von Transitions- und Demokratieforschung sowie internationaler Parteienforschung. ${ }^{4}$ Aber auch in der Praxis wurde Parteienförderung immer mehr ein Thema: Bereits Ende der 1990er Jahre zeigte Carothers die Entwicklung der „U.S. political party assistance“ im Kontext der ,general expansion of democracy assistance" (Carothers 1999: 141) auf und definierte die deutschen parteinahen Stiftungen als die „heavyweights“(141) im Bereich der internationalen Parteienförderung.

\section{Die Welt der Parteienförderer: Wer sind die internationalen Akteure und wie sehen ihre Ansätze aus? Wo positionieren sich die US-amerikanischen Organisationen?}

Die Welt der Parteienförderer lässt sich unterteilen in parteinahe Stiftungen, parteinahe Institute, Mehrparteieninstitute und Parteienbündnisse sowie internationale und intergouvernementale Organisationen (vgl. Übersicht 1).

3 Siehe beispielsweise Merkel 1997, von Beyme 1997, Morlino 1995, Randall/Svåsand 2002, Emminghaus 2003, van de Walle 2003, Burnell 2004, Randall 2007 sowie Weissenbach 2010.

4 Ausführlicher hierzu Burnell/Gerrits 2010. 


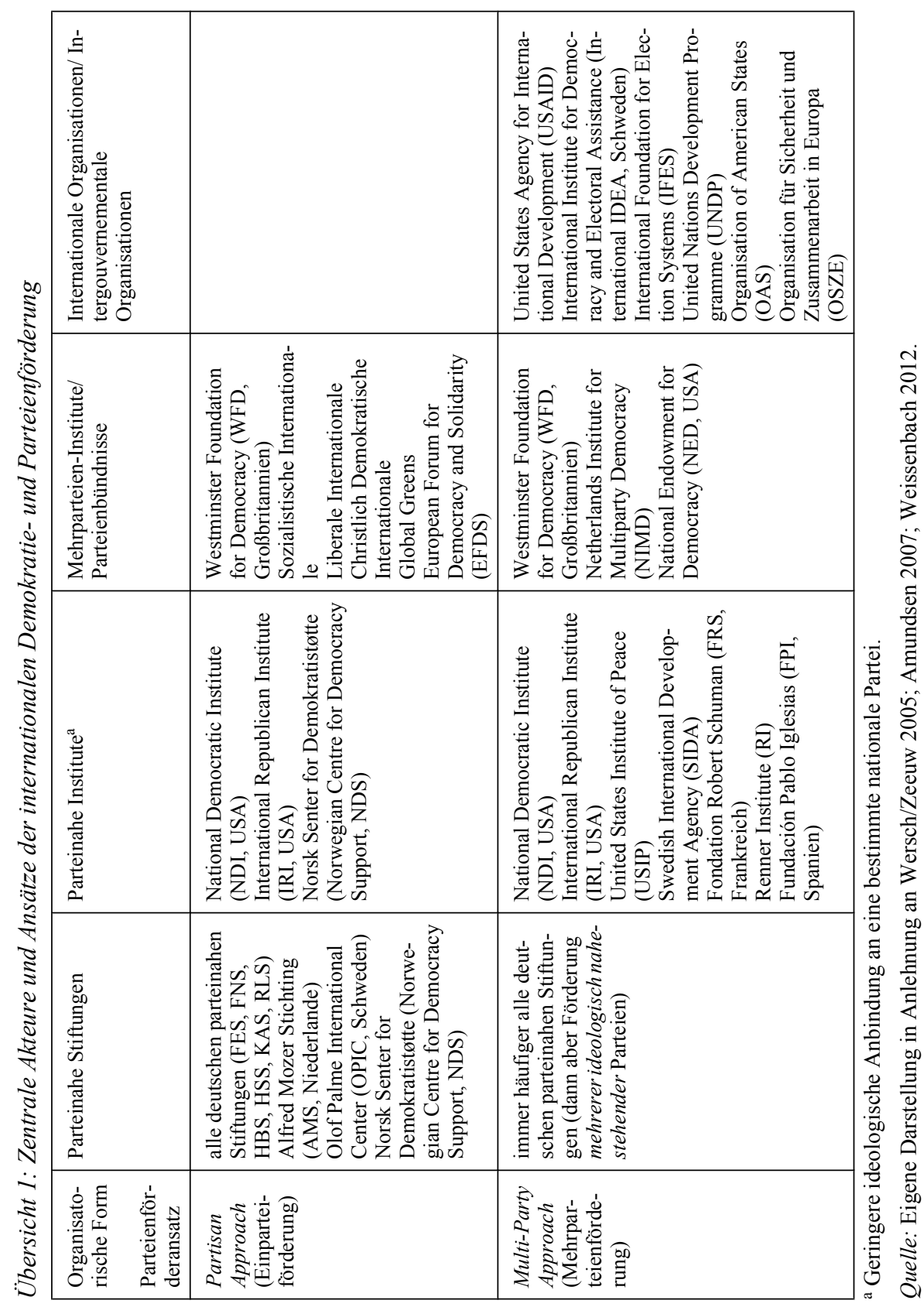


Zentrale Akteure der US-Parteienförderung wie das NDI oder das IRI gründeten sich als Nichtregierungsorganisationen, aber de facto parteinahe Institute, und knüpften in ihrer Struktur und ihren Förderzielen an das Vorbild der deutschen parteinahen Stiftungen an. Gemeinsam ist ihnen das Ziel ihrer Arbeit in Transitionsstaaten: die Installierung eines demokratischen Systems und die Institutionalisierung demokratischer Parteien. ${ }^{5}$ Der Weg der Förderung - die konkreten Ansätze und Instrumente - unterscheidet sich jedoch zwischen den deutschen und den amerikanischen Akteuren: Im Gegensatz zu den deutschen parteinahen Stiftungen, die traditionell häufig enge, lang andauernde, ideologische Bindungen und Kooperationen mit einer ihr ideologisch nahestehenden Partnerpartei pflegen (Partisan Approach), ${ }^{6}$ definieren sich die meisten US-Institute sowie multilateralen und internationalen Organisationen über einen überparteilichen, eher kurzfristigen Förderansatz mit mehreren Parteien (Multi-Party Approach). ${ }^{7}$

\section{Das Spannungsfeld parteiliche Förderung versus Mehrparteienansatz}

Auf dem Kontinuum von Einparteiförderung zu Mehrparteienförderung lassen sich verschiedene Förderansätze ausdifferenzieren: ${ }^{8}$ der Partisan Approach, der MultiParty Aproach, Cross-Party Dialogues, der Institutional Approach, die International Cross-Party Collaboration und der Civil-Society Approach. Während die deutschen parteinahen Stiftungen traditionell eine ihnen ideologisch nahestehende (Schwester-)Partei über einen langen Zeitraum vor Ort aus einem ihrer Länderbüros heraus begleiten und unterstützen (Partisan Approach), ${ }^{9}$ engagieren sich die USAkteure der Parteienförderung vorrangig im Bereich der institutionellen Förderung mehrerer Parteien (Multi-Party Approach), finanzieren - wie eingangs am Beispiel des Engagements des USIP bei der Beratung syrischer oppositioneller Kräfte erwähnt - über- oder zwischenparteiliche Dialoge (Cross-Party Dialogues) oder unterstützen den Aufbau institutioneller Rahmenbedingungen (Institutional Approach).

5 Vergleiche Interviews der Verfasserin mit Mitarbeitern der deutschen parteinahen Stiftungen. Siehe dazu auch NDI 2003 und 2012 sowie IRI 2012.

6 Nach Negativerfahrungen mit der reinen Einparteiförderung in Transitionsstaaten vor allem in SubSahara Afrika in den 1970er Jahren öffnen sich die deutschen parteinahen Stiftungen jedoch immer stärker der parallelen Förderung mehrerer im Parlament vertretener Parteien. Aber auch diese Förderung verläuft mit mehreren, der jeweiligen Stiftung ideologisch nahestehenden oder ideologisch noch nicht festgelegten Parteien. Ausführlicher hierzu Erdmann 2005 sowie Weissenbach 2012.

7 Wobei die parteinahen Institute NDI und IRI sich zwischen dem Partisan Approach und dem MultiParty Approach positionieren.

8 Ausführlicher Burnell 2004 und 2005, Erdmann 2004 sowie Weissenbach 2010 und 2012.

9 Inzwischen aber die gesamte Klaviatur der Parteienförderansätze spielen. 
Das Spannungsfeld zwischen dem Ansatz der parteilichen Förderung und des Mehrparteienansatzes wurde in den letzten zehn Jahren in der Demokratieförderdebatte immer wieder diskutiert. Nicht aber die Frage nach dem besseren Förderansatz, sondern nach dem Zeitpunkt seiner Anwendung - so das zentrale Argument dieses Beitrags - ist entscheidend. Die Durchführung der beiden Ansätze eignet sich nicht zu jedem Zeitpunkt des Transitionsprozesses. Nur in bestimmten Zeitphasen befördern sie die Institutionalisierung der Parteien und deren Konsolidierungsfunktionen.

Der Partisan Approach basiert auf einer meist langjährigen Kooperation vor dem Hintergrund einer ähnlichen Werteorientierung. Aufgrund dieser engen ideologischen Zusammenarbeit ist er der in der Demokratisierungsforschung am meisten diskutierte Ansatz. Voraussetzung seiner Durchführung ist ein enormes Vorwissen über die Partnerpartei hinsichtlich ihrer Institutionalisierung, die Fähigkeit, sie in programmatischer Hinsicht und bezüglich ihrer Positionierung gegenüber demokratischen Prinzipien eindeutig einschätzen zu können, sowie eine gegenseitige gute Vertrauensbasis. Er eignet sich daher vor allem in fortgeschrittenen Phasen der demokratischen Transition (das heißt der Demokratisierung- oder Konsolidierungsphase).

Der Multi-Party Approach zielt hingegen auf die gleichwertige Unterstützung aller (oder einer Vielzahl) der im Parlament vertretenen Parteien, unabhängig von ihrer politischen Ausrichtung. Einerseits wird damit gerade in frühen Phasen des Transitionsprozesses die Netzwerkbildung zwischen sich formierenden (oder bereits bestehenden) Parteien oder oppositionellen Bewegungen unterstützt. Bis zu einem gewissen Grad erscheint der Ansatz allerdings auch in der vorangeschrittenen Konsolidierungsphase einer jungen Demokratie von Relevanz, denn im Kontext der Verhaltenskonsolidierung (Merkel 1997) geht es darum, die Bandbreite der im Parlament vertretenen Parteien dahingehend zu beraten, dass sie die Spielregeln der neuen Institutionen annehmen und ihre Willensbildungs- und Entscheidungsprozesse innerhalb dieser vollziehen.

\section{Kurzfristige Ansätze und Parteienfördertourismus: Die Förderpraxis der US-Akteure}

„Democracy promoters are not only action-oriented, they are also often infused with a certain missionary spirit, a belief that their work is by nature valuable and good, which also inhibits critical self-reflection" (Carothers 2006: 15). Während in der Parteienförderungsdebatte der letzten zehn Jahre das Spannungsfeld zwischen den aufgezeigten Ansätzen der parteilichen (Ein-)Parteiförderung und der Mehrpartei- 
enförderung, ihre Vor- und Nachteile sowie ihr Einfluss auf die demokratische Transition diskutiert wurden (Mair 2004, Carothers 2006), scheinen die Akteure selbst ihre Förderpraxis hingegen weniger kritisch zu reflektieren. Der Mehrparteienansatz der meisten US-Organisationen wird auf den ersten Blick zwar weniger stark als Einmischung in innenpolitische Belange wahrgenommen; ebenso weisen die Fördermaßnahmen der US-Akteure meist klar formulierte und festgelegte Ziele und Leitlinien auf. Ihre Ansätze orientieren sich jedoch nicht, wie etwa oben skizziert, am Verlauf der Transition. Bei ihren starren Richtlinien - so die zentrale Kritik an der Förderpraxis von US-Akteuren wie dem NDI oder dem IRI - übersehen die Förderer die Prozesshaftigkeit der länderspezifischen demokratischen Transition und die unterschiedlichen Ausprägungen, die eine junge Partei im Prozess ihrer Entwicklung und Institutionalisierung vorweist. Statische Modelle der Parteienförderung müssen vor diesem Hintergrund jedoch ins Leere laufen. Denn gerade bei unsteten Verläufen des Transitionsprozesses und sich wandelnden Parteistrukturen - wie sie in jungen Demokratien die Regel sind - sind Parteienförderer auf theoretische Leitbilder und daran ausgerichtete länderspezifische Vorstudien angewiesen, um beim Einstieg in das Feld der Parteienförderung, beziehungsweise zum Zeitpunkt der Veränderung der externen Rahmenbedingungen, in der Ziel-, Ansatz- und Maßnahmendefinition nicht ad hoc reagieren zu müssen. Solche Leitbilder finden bislang jedoch kaum Eingang in die Parteienförderpraxis der US-Institute. ${ }^{10}$ Damit ist es ihnen nicht möglich, bei einer Veränderung der länderspezifischen Rahmenbedingungen oder des Institutionalisierungslevels einer Partei vorausschauend einordnen zu können, welche Rolle politische Parteien und politische Akteure in der neuen Phase spielen.

Um solche vorausschauenden Fördermaßnahmen umsetzen zu können reicht es - wie in der US-Förderung die Regel - nicht aus, kurzfristig zu Wahlzeiten in ein Land einzufliegen und das Campaigning mehrerer, ausgewählter Parteien zu unterstützen. Ebenso wenig führt es zu einer tieferen Institutionalisierung, wenn aus der Ferne die Mieten von Parteizentralen finanziert werden, vor Ort aber keine langfristige Kooperation aufgebaut wird, wie es im Rahmen der Zusammenarbeit des US-amerikanische NDI mit der kenianischen Democratic Party der Fall ist. ${ }^{11}$ Mit den Worten eines kenianischen Oppositionspolitikers lässt sich diese Praxis der kurzfristigen Förderung als Parteienfördertourismus ${ }^{12}$ auf den Punkt bringen: Es fehlt hier an länderspezifischen Vorstudien und an langfristigen, nachhaltigen Netz-

10 Gleiches gilt allerdings häufig auch für andere Akteure wie die deutschen parteinahen Stiftungen.

11 Vergleiche Interviews der Verfasserin mit dem Generalsekretär der kenianischen Democratic Party.

12 Vergleiche Interviews der Verfasserin mit US-geförderten Parteipolitikern. 
werken zu den geförderten Parteien. Nur dann ist es möglich, wirklich Einblick in die individuelle Parteienentwicklung zu erlangen und die spezifischen Konsolidierungsfunktionen einer Partei in einem Transitionsland zu unterstützen.

\section{Schlussbemerkung}

Angesichts der zentralen Rolle, die politischen Parteien einerseits im Prozess der demokratischen Transition zukommt, und den oftmals instabilen und schwach institutionalisierten Parteiensystemen andererseits, stehen externe Demokratie- und Parteienförderer vor erheblichen Herausforderungen. Ohne eine Stärkung von politischen Parteien droht die Demokratisierung insgesamt zu scheitern. Die Förderung von einzelnen Parteien stellt andererseits eine unmittelbare politische Einmischung dar, die den politischen Wettbewerb, der ursprünglich von außen gestärkt werden sollte, unterminiert. Vor diesem Hintergrund ist ein ausdifferenziertes Förderinstrumentarium notwendig, mit dem dieser Balanceakt einem sich schnell verändernden politischen Umfeld stets angepasst werden kann. Dieser Balanceakt ist den US-Organisationen mit ihrem kurzfristigen Mehrparteienansatz bislang nicht gelungen. Erst vor dem Hintergrund von länderspezifischen Vorstudien und langfristig angelegten Fördermaßnahmen und Partnerschaften werden sie auf die unterschiedlichen Geschwindigkeiten, Stagnationen oder Rückschläge von Transitionsverläufen und Parteienentwicklung reagieren können.

\section{Literatur}

Amundson, Inge, 2007: Donor Support to Political Parties. Status and Principles, CMI Reports 6/2007.

Beyme, Claus von, 1997: Parteien im Prozeß der demokratischen Konsolidierung, in: Wolfgang Merkel/Eberhard Sandschneider (Hrsg.), Systemwechsel. Band 3: Parteien im Transformationsprozeß, Opladen, 23-56.

Burnell, Peter, 2004: Building Better Democracies. Why Political Parties Matter, London.

Burnell, Peter, 2005: Political Strategies of External Support for Democratization, in: Foreign Policy Analysis 1 (3), 361-384.

Burnell, Peter, 2006: Globalising Democracy: Party Politics in Emerging Democracies, London.

Burnell, Peter/Gerrits, André, 2010: Promoting party politics in emerging democracies, in: Democratization 17 (6), 1065-1084.

Carothers, Thomas, 1999: Aiding Democracy Abroad. The Learning Curve, Washington D. C. 
Carothers, Thomas, 2002: The end of the Transition Paradigm, in: Journal of Democracy 13 (1), 5-21.

Carothers, Thomas, 2006: Confronting the Weakest Link, Washington D.C.

Emminghaus, Christoph, 2003: Politische Parteien im Demokratisierungsprozess. Strukturen und Funktionen afrikanischer Parteiensysteme, Opladen.

Erdmann, Gero, 2005: Hesitant bedfellows: The German Stiftungen and party aid in Africa. An attempt at an Assessment, CSRG Working Paper No. 184/05, Centre for the Study of Globalisation and Regionalisation, University of Warwick.

International Republican Institute (IRI), 2012: Political Party Development, http:// www.iri.org/discover-our-mission-0 (Stand: 20.7.2012).

Lau, Jörg, 2012: Das neue Syrien kommt aus Wilmersdorf, Die Zeit, 26.7.2012, 2. Mair, Stefan, 2004: Multi-Partisan or Bi-Partisan Co-operation. What is the Best Solution for Democracy Assistance?, in: European Profile in Democracy Support. Conference Reader 'Enhancing the European Profile in Democracy Assistance', The Hague, July 5-6, Democracy AGENDA: Alliance for Generating a European Network for Democracy Assistance.

Merkel, Wolfgang, 1997: Parteien und Parteiensysteme im Transformationsprozess: ein interregionaler Vergleich, in: Wolfgang Merkel/Eberhard Sandschneider (Hrsg.), Systemwechsel. Band 3: Parteien im Transformationsprozess, Opladen, 337-371.

Morlino, Leonardo, 1995: Political Parties and Democratic Consolidation in Southern Europe, in: Richard Gunther/ Nikiforos P. Diamondourus/Hans-Jürgen Puhle (Hrsg.), The Politics of Democratic Consolidation. Southern Europe in Comparative Perspective, Baltimore, 315-388.

National Democratic Institute (NDI), 2003: A Guide to Political Party Development, Washington.

National Democratic Institute (NDI), 2012: Political Parties, http://www.ndi.org/ content/political-parties (Stand: 20.7.2012).

ohne Autor (o. A.), 2012: Prozess gegen Stiftungsmitarbeiter in Ägypten vertagt, http://www.tagesschau.de/ausland/prozessstiftungenkairo100.html (Stand: 20.7.2012).

Randall, Vicky, 2007: Political Parties and Democratic Development States, in: Development Policy Review 25 (5), 633-652.

Randall, Vicky/Svåsand, Lars, 2002: Party institutionalization in new democracies, in: Party Politics 8 (1), 5-29.

Walle, Nicolas van de, 2003: Presidentialsim and Clientelism in Africa's Emerging Party Systems, in: Journal of Modern African Studies 41 (2), 297-321. 
Wegerhoff, Cornelia, 2012: Ägyptische Führung wittert ausländische Verschwörung, http://www.tagesschau.de/ausland/aegyptendurchsuchungen112.html (Stand: 20.7.2012).

Weissenbach, Kristina, 2010: Political party assistance in transition: the German ,Stiftungen' in sub-Saharan Africa, in: Democratization 17 (6), 1225-1249.

Weissenbach, Kristina, 2012: Parteienförderung im Transitionsprozess, Wiesbaden (im Erscheinen).

Wersch, Jos van/De Zeeuw, Jeroen, 2005: Mapping European Democracy Assistance, Clingendal Working Paper 36, Den Haag.

Korrespondenzanschrift:

Dr. des. Kristina Weissenbach

Institut für Politikwissenschaft

Universität Duisburg-Essen

Lotharstraße 65, LF 312

47057 Duisburg

E-Mail: kristina.weissenbach@uni-due.de

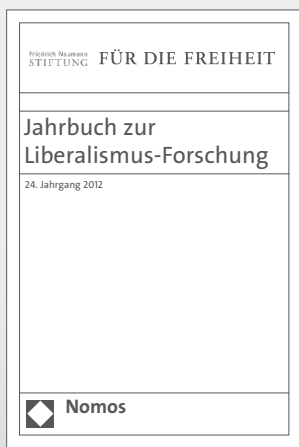

\section{Jahrbuch zur Liberalismus-Forschung}

24. Jahrgang 2012

Herausgegeben von Eckart Conze, Joachim Scholtyseck,

Erich Weede, in Verbindung mit Jürgen Frölich und

Ewald Grothe

im Auftrag der Friedrich-Naumann-Stiftung

für die Freiheit

2012, 274 S., brosch., 39,- €, ISBN 978-3-8329-7692-7

Weitere Informationen: www.nomos-shop.de/19479

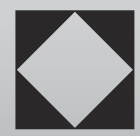

